\title{
HAT-P-11: Discovery of a Second Planet and a Clue to Understanding Exoplanet Obliquities
}

\author{
Samuel W. Yee ${ }^{1}$ (10), Erik A. Petigura ${ }^{1,7}$ (D), Benjamin J. Fulton ${ }^{1,8}$ (10), Heather A. Knutson ${ }^{1}$, Konstantin Batygin ${ }^{1}$, \\ Gáspár Á. Bakos ${ }^{2,9}$ (D), Joel D. Hartman ${ }^{2}$ (D) , Lea A. Hirsch ${ }^{3}$ (D), Andrew W. Howard ${ }^{1}$ (D), Howard Isaacson ${ }^{3}$ (D), \\ Molly R. Kosiarek ${ }^{4,10}$ (iD), Evan Sinukoff ${ }^{1,5}$ (iD), and Lauren M. Weiss ${ }^{6,11}$ (D) \\ ${ }^{1}$ California Institute of Technology, Pasadena, CA, 91125, USA; syee@ caltech.edu \\ ${ }^{2}$ Department of Astrophysical Sciences, Princeton University, Princeton, NJ 08544, USA \\ ${ }^{3}$ University of California, Berkeley, Berkeley, CA 94720, USA \\ ${ }^{4}$ University of California, Santa Cruz, Santa Cruz, CA, 95064, USA \\ ${ }^{5}$ Institute for Astronomy, University of Hawai $i$, Honolulu, HI 96822, USA \\ ${ }^{6}$ University of Montréal, Montréal, QC H3T 1J4, Canada \\ Received 2017 November 9; revised 2018 April 3; accepted 2018 April 17; published 2018 May 25
}

\begin{abstract}
HAT-P-11 is a mid-K dwarf that hosts one of the first Neptune-sized planets found outside the solar system. The orbit of HAT-P-11b is misaligned with the star's spin—one of the few known cases of a misaligned planet orbiting a star less massive than the Sun. We find an additional planet in the system based on a decade of precision radial velocity (RV) measurements from Keck/High Resolution Echelle Spectrometer. HAT-P-11c is similar to Jupiter in its mass $\left(M_{P} \sin i=1.6 \pm 0.1 M_{J}\right)$ and orbital period $\left(P=9.3_{-0.5}^{+1.0}\right.$ year $)$, but has a much more eccentric orbit $(e=0.60 \pm 0.03)$. In our joint modeling of RV and stellar activity, we found an activity-induced RV signal of $\sim 7 \mathrm{~m} \mathrm{~s}^{-1}$, consistent with other active $\mathrm{K}$ dwarfs, but significantly smaller than the $31 \mathrm{~m} \mathrm{~s}^{-1}$ reflex motion due to HAT-P-11c. We investigated the dynamical coupling between HAT-P-11b and $\mathrm{c}$ as a possible explanation for HAT-P-11b's misaligned orbit, finding that planet-planet Kozai interactions cannot tilt planet b's orbit due to general relativistic precession; however, nodal precession operating on million year timescales is a viable mechanism to explain HAT-P-11b's high obliquity. This leaves open the question of why HAT-P-11c may have such a tilted orbit. At a distance of $38 \mathrm{pc}$, the HAT-P-11 system offers rich opportunities for further exoplanet characterization through astrometry and direct imaging.
\end{abstract}

Key words: planetary systems - planets and satellites: detection - planets and satellites: dynamical evolution and stability - stars: individual (HAT-P-11)

Supporting material: machine-readable table

\section{Introduction}

HAT-P-11 is a mid-K dwarf known to host HAT-P-11b, a super-Neptune on a $P=4.88$ day orbit, with $M_{P}=23.4 \pm 1.5 M_{\oplus}$ and $R_{P}=4.36 \pm 0.06 R_{\oplus}$. The planet was first discovered by Bakos et al. (2010) using ground-based photometry and confirmed by radial velocities (RVs), which constrained its mass and eccentricity. Bakos et al. (2010) found a moderate eccentricity of $e=0.198 \pm 0.046$, the first clue that the HAT-P-11 system is dynamically hot. At the time, HAT-P-11b was the smallest planet discovered by ground-based transit photometry.

HAT-P-11 was observed by the Kepler Space Telescope (Borucki et al. 2010) during its prime mission (2009-2013). Deming et al. (2011) and Sanchis-Ojeda \& Winn (2011) analyzed this data and found spot-crossing anomalies at particular phases of the transit of HAT-P-11b, which are consistent with a nearly polar orbit crossing two active latitudes on the host star. This was in agreement with the results from two independent RV campaigns by Winn et al. (2010b) and Hirano et al. (2011), who used the Rossiter-McLaughlin (RM) effect to measure the planet's orbital obliquity to be $\lambda \approx 100^{\circ}$. Using the Kepler photometry, Huber et al. (2017) also reported a tentative detection of HAT-P-11b's secondary eclipse.

\footnotetext{
${ }^{7}$ Hubble Fellow.

8 Texaco Postdoctoral Fellow.

9 Packard Fellow.

${ }^{10}$ NSF Graduate Research Fellow.

11 Trottier Fellow.
}

Here, we present an extended RV time series spanning 10 years (Section 2), which shows a long-period Keplerian signal with $P \approx 9$ years. While HAT-P-11 is chromospherically active, we show in Section 3 that the RV signal cannot be explained by activity alone. In Section 4, we model the RV time series including the effects of planet $b$, planet $c$, and stellar activity. We investigate the dynamical connection between the two planets in Section 5 and find that HAT-P-11c can explain the high obliquity of HAT-P-11b. Finally, we place the HATP-11 system in context of other exoplanet systems (Section 6) and discuss prospects for future characterization (Section 7).

\section{Spectroscopic Observations}

The California Planet Search (CPS; Howard et al. 2010) has observed the HAT-P-11 system since 2007 August with the High Resolution Echelle Spectrometer (HIRES; Vogt et al. 1994) at the Keck I 10 m telescope on Maunakea. We collected a total of 253 spectra with an iodine cell in front of the spectrometer, which imprints iodine absorption lines to serve as a wavelength reference against which RVs can be measured precisely. The spectra have signal-to-noise ratios between 100 and 130 per pixel on blaze near $5500 \AA$.

\subsection{Radial Velocities}

We used the standard CPS pipeline described in Howard et al. (2010) to determine the RVs. This involves forward modeling the stellar and iodine spectra convolved with the instrumental 

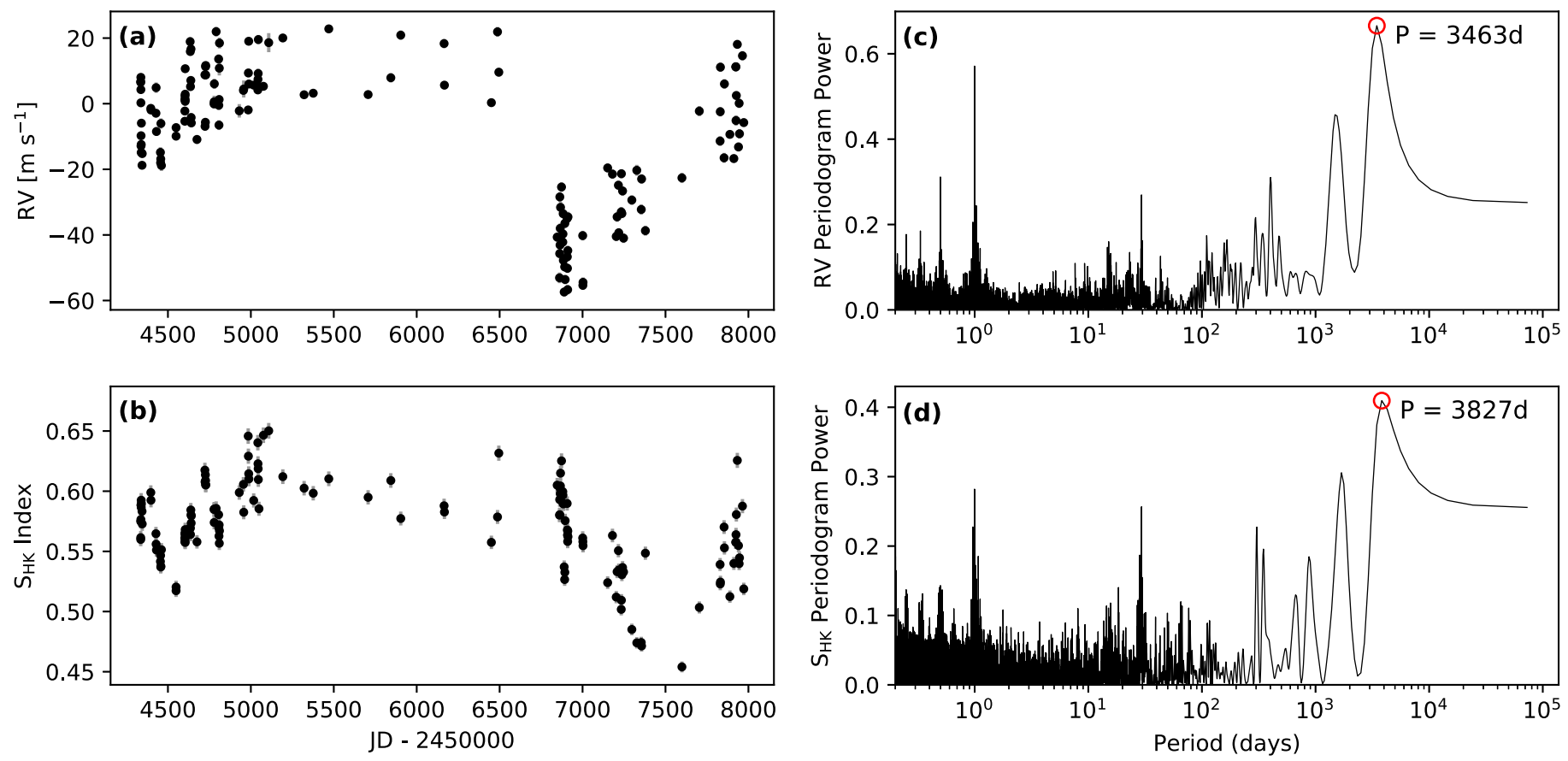

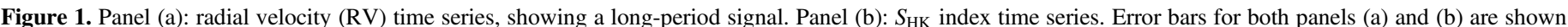

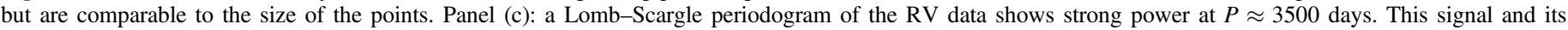

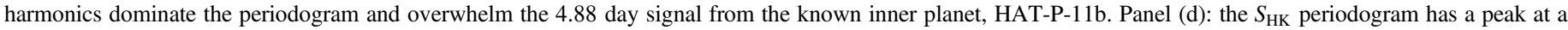
similar period. We note the strong signal in both periodograms at 29 days, the inferred rotational period of HAT-P-11.

Table 1

Radial Velocity and Activity Measurements

\begin{tabular}{|c|c|c|c|c|c|c|c|}
\hline $\begin{array}{l}\text { Time } \\
\text { BJD }_{\mathrm{TBD}}\end{array}$ & $\begin{array}{c}\mathrm{RV} \\
\left(\mathrm{m} \mathrm{s}^{-1}\right)\end{array}$ & $\begin{array}{c}\sigma(\mathrm{RV}) \\
\left(\mathrm{m} \mathrm{s}^{-1}\right)\end{array}$ & $S_{\mathrm{HK}}$ Index & $\sigma\left(S_{\mathrm{HK}}\right)$ & $\mathrm{H} \alpha$ Index & $\sigma(\mathrm{H} \alpha)$ & Flag \\
\hline 2454335.891030 & 6.50 & 1.03 & 0.5599 & 0.0056 & 0.04539 & 0.00026 & 1 \\
\hline 2454335.897680 & 6.75 & 1.09 & 0.5614 & 0.0056 & 0.04537 & 0.00026 & 1 \\
\hline 2454336.746470 & 8.03 & 0.94 & 0.5748 & 0.0057 & 0.04533 & 0.00025 & 1 \\
\hline 2454336.859340 & 4.30 & 1.03 & 0.5751 & 0.0058 & 0.04531 & 0.00026 & 1 \\
\hline 2454336.947330 & 0.27 & 1.00 & 0.5765 & 0.0058 & 0.04543 & 0.00027 & 1 \\
\hline 2454337.729220 & -12.86 & 1.14 & 0.5886 & 0.0059 & 0.04602 & 0.00028 & 1 \\
\hline
\end{tabular}

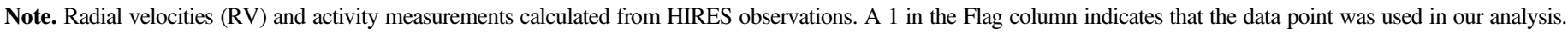
(This table is available in its entirety in machine-readable form.)

point-spread function for different spectral segments (Marcy \& Butler 1992; Valenti et al. 1995). The complete set of RV data is presented in Table 1, with a median uncertainty of $1.4 \mathrm{~m} \mathrm{~s}^{-1}$. In the subsequent analysis, we have excluded two sets of very high cadence observations taken within $4 \mathrm{hr}$ of the transit of HAT-P$11 \mathrm{~b}$, which are affected by the RM effect.

This leaves us with 144 remaining RV measurements, which are plotted in Figure 1(a). In their original discovery, Bakos et al. (2010) reported a significant long-term drift over two years of RV observations, which they interpreted as a possible second planet. With our extended observational baseline of 10 years, we see that this long-period trend has reversed, suggesting that we have now viewed a complete orbit of this outer companion. A generalized Lomb-Scargle periodogram (Zechmeister and Kürster 2009) of the raw RVs shows a peak at $\sim 3463$ days (Figure 1(c)), just over 9 years.

\subsection{Stellar Activity Indicators}

HAT-P-11 is known to be a spotted, chromospherically active star (Deming et al. 2011; Morris et al. 2017a). Stellar activity can produce spurious RV signals that may be mistaken for a planet (see, e.g., Haywood et al. 2014; Robertson et al. 2014). To investigate whether stellar activity could account for the nine-year RV signal, we extract two activity indices from our spectroscopic observations.

The Mount Wilson $S_{\mathrm{HK}}$ index traces the chromospheric emission in the cores of the Ca II $\mathrm{H} \& \mathrm{~K}$ lines (Vaughan et al. 1978) and is a standard activity tracer for main-sequence stars. We extract $S_{\mathrm{HK}}$ from our spectra following the procedure of Isaacson \& Fischer (2010), and our measurements are precise to $1 \%$.

We also measured the $\mathrm{H} \alpha$ index, which has been found to be a good activity tracer for late-type stars (Gomes da Silva et al. 2011; Robertson et al. 2014). While $\mathrm{H} \alpha$ tracked $S_{\mathrm{HK}}$ closely, the size of the variations were on the $1 \%-2 \%$ level, comparable to the measurement uncertainty. Therefore, we henceforth use $S_{\mathrm{HK}}$ as the activity tracer. Both activity indices for each observation are provided in Table 1.

The periodogram of $S_{\mathrm{HK}}$ (Figure 1(d)) has a peak at $\sim 3800$ days, close to peak found in the RV periodogram. 

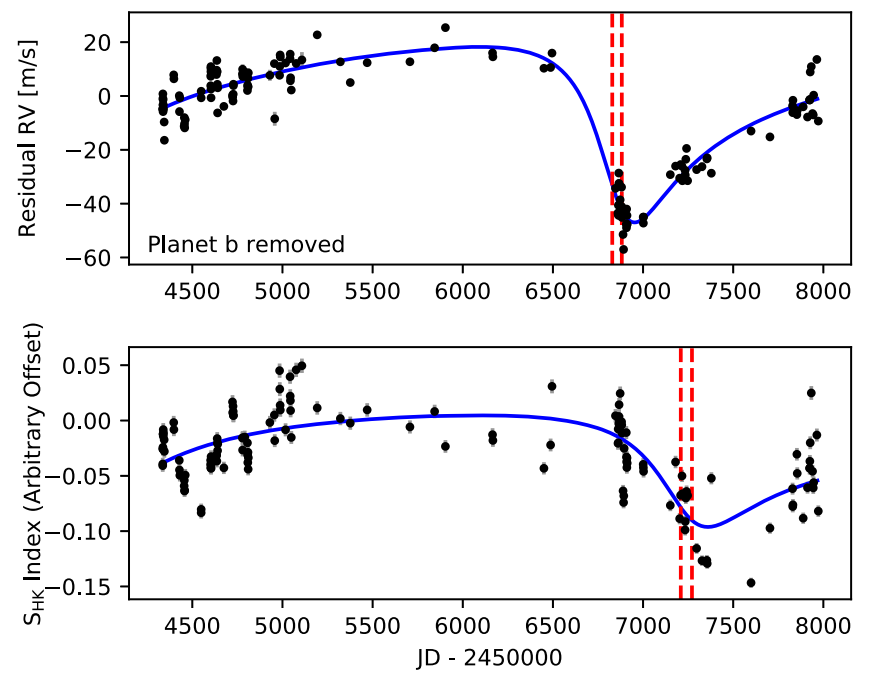

Figure 2. Top: the maximum-likelihood Keplerian model fit to the residual RVs, after removing the effect of the inner planet. Vertical dashed lines mark the $1 \sigma$ confidence interval for the time of periastron passage. Bottom: using the model parameters and uncertainties derived from the RV fit as priors, we fit a Keplerian to the $S_{\mathrm{HK}}$ indices. The time of periastron passage for this model is 412 days later, demonstrating a significant phase offset between the two signals.

Morris et al. (2017a, 2017b) also observed this activity signal and interpreted it as a solar-like dynamo. Given the comparable timescales of the RV and activity cycles, we consider whether activity could be responsible for the RV variability in the following section.

\section{Is the Long-period RV Signal Due to Stellar Activity?}

Our decade of RV observations of HAT-P-11 have revealed a long-period signal, suggestive of a planet. Here, we assess whether this signal could be caused by stellar activity. We show that activity is incompatible with the observed nine-year RV signal for three reasons: (1) the observed amplitude is much larger than activityinduced RV variability seen in similar stars, (2) there is a significant phase offset between the activity and RV cycles, and (3) the RVactivity correlation is too weak to account for the RV signal.

\subsection{Amplitude of RV Signal}

We first subtracted the effect of HAT-P-11b from the RV time series, using a model generated from the orbital parameters derived by Bakos et al. (2010). The residual RVs are shown in Figure 2 and the remaining long-period signal has a semi-amplitude of $\sim 35 \mathrm{~m} \mathrm{~s}^{-1}$.

Typical activity-induced RV signals are significantly smaller. Isaacson \& Fischer (2010) measured the chromospheric activity and RV jitter of $\sim 2600$ main-sequence and subgiant stars. For the $\sim 300$ stars in the sample similar to HAT-P-11 $(1.0<B-V<1.3)$, the typical rms in measured RVs was around $4-8 \mathrm{~m} \mathrm{~s}^{-1}$. In particular, there was no increase in jitter as a function of $S_{\mathrm{HK}}$ index, suggesting that $\mathrm{K}$ dwarfs do not have significant activity-induced jitter.

These findings were corroborated in a similar study by Lovis et al. (2011), who observed 304 FGK stars with HARPS over seven years, finding a maximum activity-induced RV signal of $11 \mathrm{~m} \mathrm{~s}^{-1}$. This study also found that RV correlation with magnetic activity is minimized in stars with $T_{\text {eff }} \approx 4800 \mathrm{~K}$, where even strong magnetic cycles induced RV signals of only several $\mathrm{m} \mathrm{s}^{-1}$.
Thus, it is unlikely that the $\sim 35 \mathrm{~m} \mathrm{~s}^{-1} \mathrm{RV}$ signal in the HAT-P-11 data could be attributed to stellar activity alone, as it is more than three times larger than previously known activityinduced signals, particularly when we consider the reduced sensitivity of RVs to chromospheric activity in $\mathrm{K}$ dwarfs.

\subsection{RV-Activity Phase Offset}

Another line of reasoning favoring the planet interpretation is the phase offset between the RV and $S_{\mathrm{HK}}$ cycles. Activityinduced RV signals arise due to suppression of convective blueshift, primarily by plages (e.g., Dumusque et al. 2014; Haywood et al. 2014). Because the $S_{\mathrm{HK}}$ index measures chromospheric $\mathrm{Ca}$ II $\mathrm{H} \& \mathrm{~K}$ emission, it is a direct measure of plage activity (Shine \& Linsky 1974). Hence, any activityinduced RV signal should move in lockstep with the $S_{\mathrm{HK}}$ activity indicator, without any phase offset.

The presence of spots may cause a phase shift between the $S_{\mathrm{HK}}$ and induced RV signal, due to masking of parts of the star that are rotationally blueshifted or redshifted (Haywood et al. 2014). The maximum offset between the two signals due to rotation is only a fraction of the stellar rotation period (29 days) and is therefore negligible when compared to the nine-year period of the RV signal.

Inspection of Figure 1 shows that the $\mathrm{RV}$ and $S_{\mathrm{HK}}$ time series reach their respective minima at times that differ by more than a year. To measure the significance of this offset, we used the publicly available RadVel software package (Fulton et al. 2018b) ${ }^{12}$ to fit a Keplerian model to the residual RVs described in Section 3.1 and to the $S_{\mathrm{HK}}$ indices (Figure 2).

For the RVs, we measured an eccentricity of $e=0.565 \pm$ 0.035 , period of $3334 \pm 220$ days, and a periastron passage of $\mathrm{JD}=2456859_{-31}^{+22}$. If this signal were in fact due to stellar activity, we would expect the shape and period of the $S_{\mathrm{HK}}$ cycle to be similar. We thus fit the $S_{\mathrm{HK}}$ time series with another Keplerian using priors on eccentricity and period corresponding to the RV fit. For the $S_{\mathrm{HK}}$ indices, we measure a "periastron passage" of JD $=2457271_{-34}^{+28}$, more than 400 days after $t_{p}$ of the RV signal. This corresponds to a phase offset of $\sim 12 \%$, a difference of $>10 \sigma$.

There is no physical basis to expect such a 400 day offset between the long-period $S_{\mathrm{HK}}$ and RV cycles. This suggests that their apparent similarity is no more than a coincidence, rather than a causative relationship between stellar activity and measured RVs.

\section{3. $R V-S_{H K}$ Correlation}

Finally, if the long-period RV variation was indeed due to stellar activity, they should be correlated across the entire data set. Figure 3 shows the residual RVs after removing the effect of planet $\mathrm{b}$ as a function of $S_{\mathrm{HK}}$ index. While there exists a weak linear correlation, the Pearson's $r$ statistic is only 0.34 , indicating that up to a third of the total rms variation in RVs can be accounted for by stellar activity. The remaining variation, reflected in the large scatter around the fitted line, must be due to another mechanism.

\subsection{Summary}

Stellar activity alone is insufficient to explain the RV variability of HAT-P-11. The amplitude of the RV variation is

\footnotetext{
${ }^{12}$ https://radvel.readthedocs.io
} 


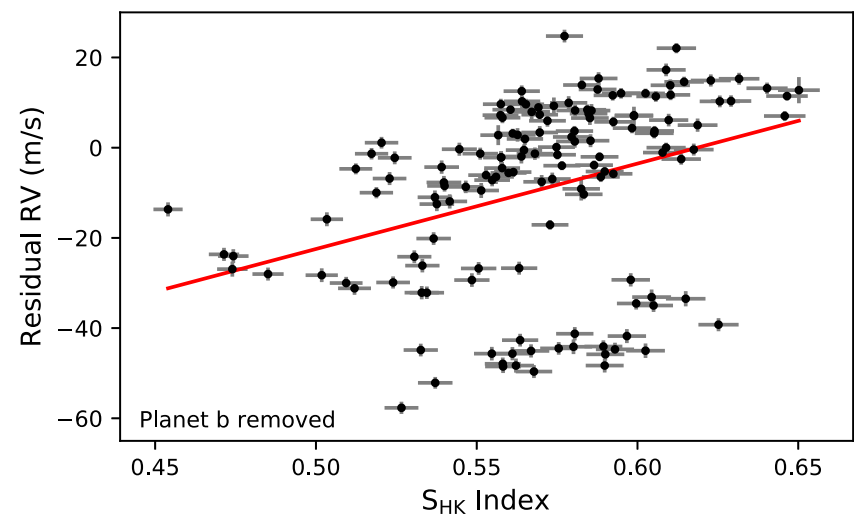

Figure 3. Residual RVs as a function of $S_{\mathrm{HK}}$ index. While there is some correlation between the RVs and stellar activity, the low Pearson's $r$ statistic suggests that only $\sim 34 \%$ of the total variation could be part of an activity-induced signal.

too large to be caused solely by stellar activity (Section 3.1), and the activity cycle is offset from the RV signal by more than a year (Section 3.2). The correlation between the residual RVs and $S_{\mathrm{HK}}$ indices also show that most of the RV variation cannot be attributed to stellar activity (Section 3.3). We therefore subsequently adopt a two-planet interpretation for the data.

\section{RV Modeling}

Here, we describe our modeling of the HAT-P-11 RVs that includes contributions from two planets. The weak RV-S $S_{\mathrm{HK}}$ correlation, described in Section 3.3, motivated an analysis that simultaneously includes the effects of stellar activity.

To better understand the connection between the residual RVs and stellar activity, we identify four seasons of $<180$ days with at least 15 observations, over which long-period variations can be neglected (Figure 4). For each of these four intervals, we find much stronger linear correlations between the $S_{\mathrm{HK}}$ index and residual $\mathrm{RVs}$ than the correlation present in the full set of observations. The Pearson's $r$ statistics were $>0.5$ for all intervals, and the $p$-values were less than $5 \%$. We also observe that the high-cadence segments have different mean RVs, but the correlations have consistent slopes as determined by a bootstrap resampling. This suggests that stellar activity has a small but consistent effect on the RV measurements, but it cannot explain the offsets between observing seasons, since they occur at the same $S_{\mathrm{HK}}$ values but have mean RVs that differ by more than $50 \mathrm{~m} \mathrm{~s}^{-1}$.

Given this short-timescale RV-activity correlation, we modeled the data using a two-planet Keplerian model as well as a linear correlation between the RVs and $S_{\mathrm{HK}}$. We used the RadVel package (Fulton et al. 2018b) to perform maximumlikelihood fitting and MCMC parameter estimation.

We fixed the period and time of conjunction for HAT-P-11b according to the values derived by Huber et al. (2017) from four years of Kepler data. The remaining orbital parameters for planets $\mathrm{b}$ and $\mathrm{c}$, as well as an average RV offset, $\gamma$, were allowed to float. We parameterized $e$ and $\omega$ of each planet as $\sqrt{e} \cos \omega$ and $\sqrt{e} \sin \omega$ to guard against a bias toward non-zero eccentricities as recommended by Eastman et al. (2013). We also imposed a beta distribution prior for the eccentricities recommended in Kipping (2013).

The slope of the activity-RV correlation is a new free parameter, $c_{S}$, such that the induced RV signal is $c_{S} \Delta S_{\mathrm{HK}, i}$.
Here, $\Delta S_{\mathrm{HK}, i} \equiv S_{\mathrm{HK}, i}-\overline{S_{\mathrm{HK}}}$ is the mean-centered $S_{\mathrm{HK}}$ index at time $t_{i}$. Any further constant offset is absorbed into $\gamma$.

The likelihood is

$\ln \mathcal{L}=-\frac{1}{2} \sum_{i}\left[\frac{\left(v_{i}-v_{m, i}-c_{S} \Delta S_{\mathrm{HK}, i}\right)^{2}}{\sigma_{i}^{2}+\sigma_{\mathrm{jit},}^{2}}+\ln 2 \pi\left(\sigma_{i}^{2}+\sigma_{\text {jit }}^{2}\right)\right]$,

where $v_{i}$ and $v_{m, i}$ are the measured and model RVs at time $t_{i}, \sigma_{i}$ is the corresponding uncertainty on the measured $\mathrm{RV}$, and $\sigma_{\mathrm{jit}}$ is the jitter.

The results of our RV fit are shown in Figure 5 and the derived planetary parameters are given in Table 2 . We also provide the posterior distributions from our MCMC analysis in Appendix C. We find that HAT-P-11c is a $M_{P} \sin i=1.60_{-0.08}^{+0.09} M_{J}$ giant planet with semimajor axis of $a=4.13_{-0.16}^{+0.29}$ au. Its high-eccentricity orbit $\left(e=0.601_{-0.031}^{+0.032}\right)$ gives it a periastron distance of $1.67_{-0.13}^{+0.14}$ au and an apoastron distance of $6.61_{-0.30}^{+0.52}$ au. This large separation reached at apoastron will have a positive effect on any future attempts to detect the planet via direct imaging, as we discuss in Section 7.

Once the effect of both planets is removed (Figure 4(d)), residual RVs show a strong linear correlation with the $S_{\mathrm{HK}}$ values (Pearson's $r=0.479, p$-value $=8 \times 10^{-9}$ ), where the offsets between the four observing seasons are eliminated. The total semi-amplitude of the activity-induced RV is $\sim 7 \mathrm{~m} \mathrm{~s}^{-1}$, consistent with that observed in stars of similar spectral types (see Section 3.1).

We also investigated models with higher and lower complexity. We first examined a single-planet model with activity as well as a two-planet model without activity correction. These models were not favored when compared using the Bayesian information criterion (BIC; Schwarz 1978). We also considered the possibility of additional planets in the system, but these were not found by a two-dimensional Keplerian Lomb-Scargle (2DKLS; O'Toole et al. 2009) periodogram search. We describe these model comparisons in detail in Appendix A.

Finally, to ensure our methodology does not always favor planets over activity, we applied an identical analysis to the HD 99492 system, another active mid-K dwarf with long-period activity and RV signals (Appendix B). In this case, the BIC rejects a planetary explanation for the RVs and prefers a pure stellar activity model, in agreement with the findings of Kane et al. (2016).

\section{System Dynamics and Spin-Orbit Misalignment}

The orbit of HAT-P-11b is known to be misaligned with its host star's spin axis, with an obliquity of $\lambda \approx 100^{\circ}$, corresponding to a nearly polar orbit (see Section 1 ). There are a number of other planets with misaligned orbits (see Albrecht et al. 2012; Dai \& Winn 2017). Many explanations have been proposed for such misalignments, including KozaiLidov cycles (e.g., Fabrycky \& Tremaine 2007), planet-planet scattering (e.g., Nagasawa et al. 2008), primordial tilting of the protoplanetary disk (e.g., Batygin 2012), or angular momentum transport by internal gravity waves (e.g., Rogers et al. 2012). Here, we examine the dynamical coupling between HAT-P-11b and $\mathrm{c}$ and assess if it can explain the observed misalignment.

The orbital angular momentum of HAT-P-11c is much greater than that of HAT-P-11b and the star's spin angular momentum, allowing us to make the approximation that the orbital plane of 

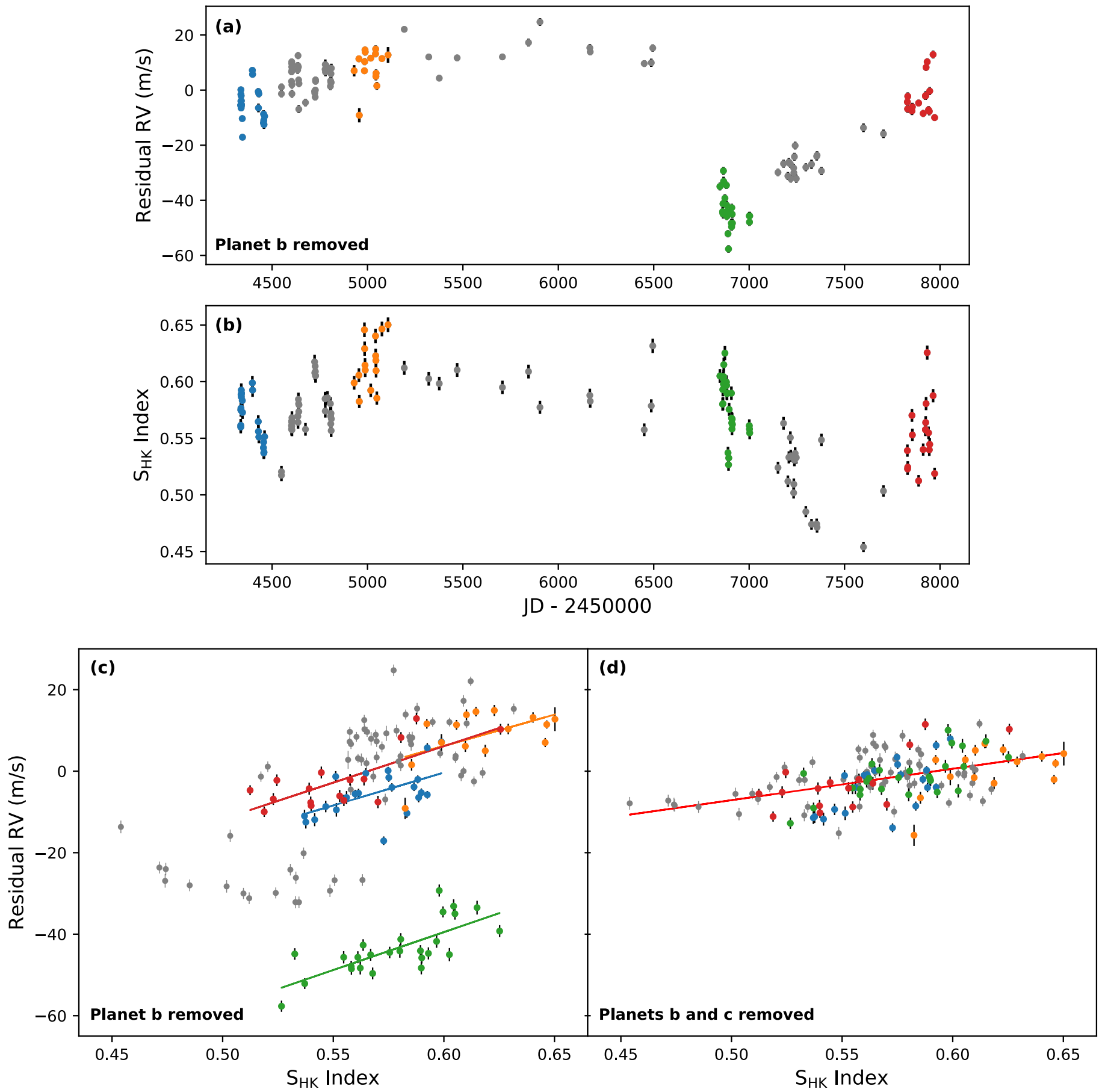

Figure 4. Panel (a): residual RVs, after removing the effect of the inner planet. We identified four different intervals of up to 180 days each with relatively high observational cadence, over which the RV signal of any putative outer planet can be neglected. These four intervals are marked with colored points. Panel (b): $S_{\mathrm{HK}}$ indices, with the same observation periods marked. Panel (c): for each identified period, a strong correlation between residual RV and $S_{\mathrm{HK}}$ can be observed, with the slope consistent over all four seasons. Panel (d): same as panel (c), but with the effects of both planets b and c removed. The offsets between the four observing seasons have vanished, such that the correlations from each season are now fully consistent with each other. For clarity, the errors in $S_{\mathrm{HK}}$ index are not shown in panels (c) and (d).

planet $\mathrm{c}$ is invariant. As a matter of convenience, we define angles that describe the orientation of planet b's orbit, inclination $i_{b}$ and argument of periastron $\omega_{b}$, with respect to the orbital plane of planet c. Note that this reference plane is not the sky plane, which is often used to describe the orbits of transiting planets. In this coordinate system, $i_{b}$ is therefore the relative inclination between the two planets. Following Mardling (2010), we write down the orbit-averaged Hamiltonian for the interaction of the two planets, 

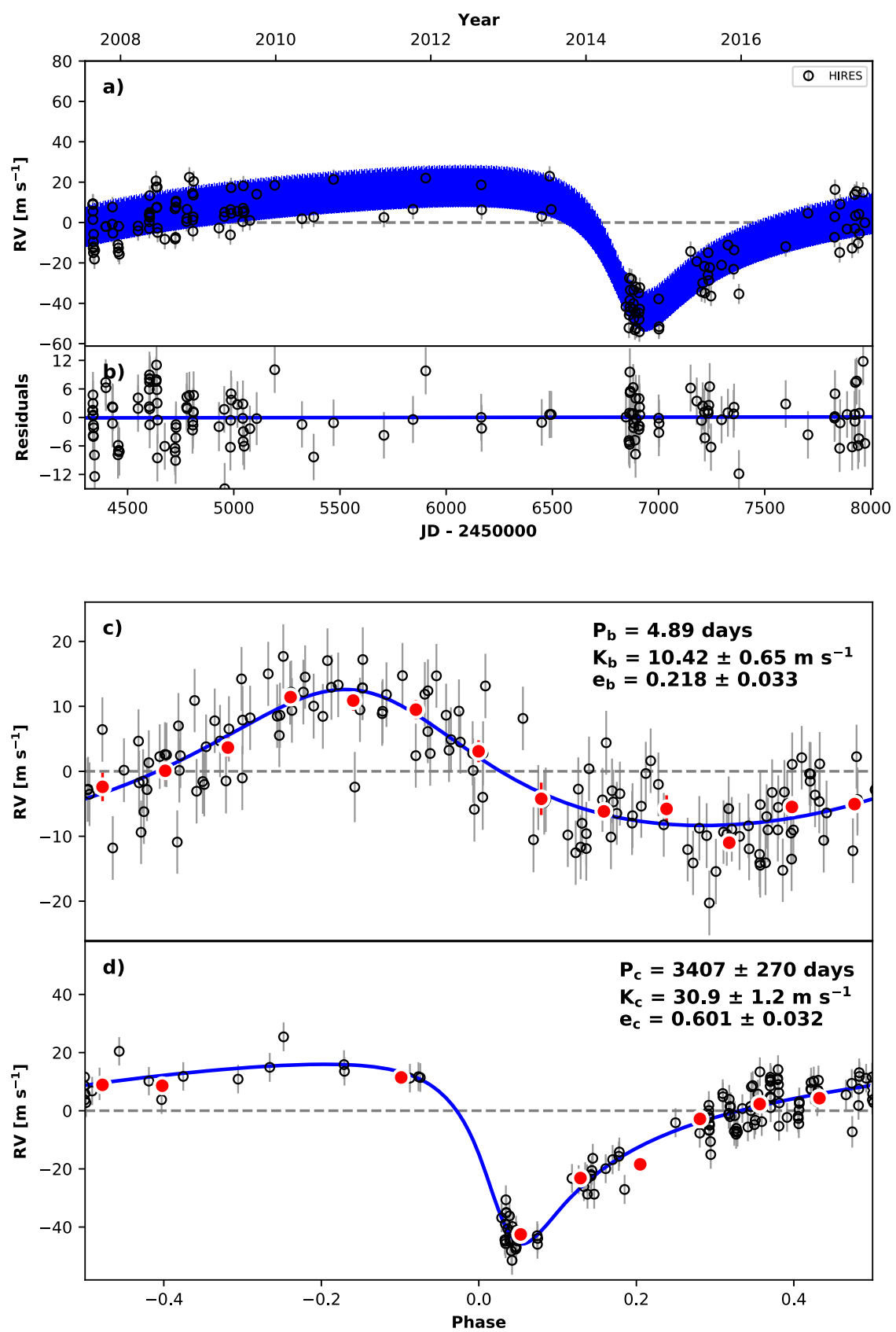

Figure 5. Two-planet Keplerian fit to the RVs, including a linear correlation between $S_{\mathrm{HK}}$ and radial velocities. Panel (a): the most probable model and full radial velocity time series. Panel (b): residuals from the most probable model, after removing the effect of both planets and the $S_{\mathrm{HK}}$ decorrelation. Panels (c) and (d): phase-folded RVs and the most probable model for planets $\mathrm{b}$ and c, respectively, with contributions of the other planet and $S_{\mathrm{HK}}$ decorrelation removed. The large red circles show phase-binned RVs.

expanded to quadrupole order in semimajor axis ratio (Kaula 1964) $^{13}$

$$
\begin{aligned}
\mathcal{H}= & \frac{1}{4} \frac{\mathcal{G} m_{b} m_{c}}{a_{c}}\left(\frac{a_{b}}{a_{c}}\right)^{2}\left(\frac{1}{\sqrt{1-e_{c}^{2}}}\right)^{3} \\
& {\left[\left(1+\frac{3}{2} e_{b}^{2}\right)\left(\frac{3}{2} \cos ^{2} i_{b}-\frac{1}{2}\right)+\frac{15}{4} e_{b}^{2} \sin ^{2} i_{b} \cos 2 \omega_{b}\right] . }
\end{aligned}
$$

The second term, containing $e_{b}^{2} \sin ^{2} i_{b}$, gives rise to the Kozai-Lidov mechanism, in which the inner planet

\footnotetext{
$\overline{13}$ The semimajor axis ratio in this sytem is $\frac{a_{b}}{a_{c}} \approx 0.01$, warranting a leadingorder truncation of the Hamiltonian.
}

undergoes cycles trading large inclinations for large eccentricities. However, because HAT-P-11b is very close in to its star, general relativistic (GR) effects cause apsidal precession, which may suppress Kozai-Lidov oscillations under suitable conditions (e.g., Ford et al. 2000; Fabrycky \& Tremaine 2007). Hence, we must include an additional GR term in the Hamiltonian.

We can write the Hamiltonian including the GR term using scaled canonical Delaunay variables, where $\Omega_{b}$ is the longitude of ascending node of planet $b$ :

$$
\begin{aligned}
& G=\sqrt{1-e_{b}^{2}} \quad g=\omega_{b} \\
& H=\sqrt{1-e_{b}^{2}} \cos i_{b} \quad h=\Omega_{b}
\end{aligned}
$$


Table 2

System Parameters

\begin{tabular}{lcc}
\hline \hline Stellar Parameters & & \\
\hline$R_{\star}\left(R_{\odot}\right)$ & $0.683 \pm 0.009$ & $\mathrm{~A}$ \\
$M_{\star}\left(M_{\odot}\right)$ & $0.809_{-0.03}^{+0.02}$ & $\mathrm{~B}$ \\
$T_{\text {eff }}(\mathrm{K})$ & $4780 \pm 50$ & $\mathrm{~B}$ \\
{$[\mathrm{Fe} / \mathrm{H}]$} & $+0.31 \pm 0.05$ & $\mathrm{~B}$ \\
$V(\mathrm{mag})$ & $6.57 \pm 0.09$ & $\mathrm{~B}$ \\
$v \sin i\left(\mathrm{~km} \mathrm{~s}^{-1}\right)$ & $1.5 \pm 1.5$ & $\mathrm{~B}$ \\
$P_{\text {rot }}(\mathrm{d})$ & 29.2 & $\mathrm{~B}$ \\
Age $(\mathrm{Gyr})$ & $6.5_{-4.1}^{+5.9}$ & $\mathrm{~B}$ \\
Distance $(\mathrm{pc})$ & $37.89 \pm 0.33$ & $\mathrm{C}$ \\
\hline
\end{tabular}

Planetary Parameters

Planet b

\begin{tabular}{lcc}
\hline$P$ (days) & $\equiv 4.887802443$ & $\mathrm{D}$ \\
$T_{\text {conj }}(\mathrm{JD})$ & $\equiv 2454957.8132067$ & $\mathrm{D}$ \\
$e$ & $0.218_{-0.031}^{+0.034}$ & $\mathrm{E}$ \\
$\omega\left(^{\circ}\right)$ & $19_{-16}^{+14}$ & $\mathrm{E}$ \\
$M_{P} \sin i\left(M_{\oplus}\right)$ & $23.4 \pm 1.5$ & $\mathrm{E}$ \\
$a(\mathrm{au})$ & $0.05254_{-0.00066}^{+0.00064}$ & $\mathrm{E}$ \\
$R_{\mathrm{P}}\left(R_{\oplus}\right)$ & $4.36 \pm 0.06$ & $\mathrm{D}$ \\
$r_{\text {peri }}(\mathrm{au})$ & $0.0413_{-0.0019}^{+0.0018}$ & $\mathrm{E}$ \\
$T_{\text {peri }}(\mathrm{JD})$ & $2454957.15_{-0.20}^{+0.17}$ & $\mathrm{E}$ \\
$r_{\text {apo }}(\mathrm{au})$ & $0.0637_{-0.0019}^{+0.0020}$ & $\mathrm{E}$ \\
$T_{\text {apo }}(\mathrm{JD})$ & $2454959.60_{-0.20}^{+0.17}$ & $\mathrm{E}$ \\
\hline
\end{tabular}

Planet c

\begin{tabular}{lcc}
\hline$P$ (days) & $3407_{-190}^{+360}$ & $\mathrm{E}$ \\
$T_{\text {conj }}(\mathrm{JD})$ & $2456746_{-32}^{+24}$ & $\mathrm{E}$ \\
$e$ & $0.601_{-0.031}^{+0.032}$ & $\mathrm{E}$ \\
$\omega\left(^{\circ}\right)$ & $143.7_{-4.9}^{+4.8}$ & $\mathrm{E}$ \\
$M_{P} \sin i\left(M_{\oplus}\right)$ & $507_{-27}^{+30}$ & $\mathrm{E}$ \\
$a(\mathrm{au})$ & $4.13_{-0.16}^{+0.29}$ & $\mathrm{E}$ \\
$r_{\text {peri }}(\mathrm{au})$ & $1.67_{-0.13}^{+0.14}$ & $\mathrm{E}$ \\
$T_{\text {peri }}(\mathrm{JD})$ & $2456862_{-26}^{+20}$ & $\mathrm{E}$ \\
$r_{\text {apo }}(\mathrm{au})$ & $6.61_{-0.30}^{+0.52}$ & $\mathrm{E}$ \\
$T_{\text {apo }}(\mathrm{JD})$ & $2458565_{-87}^{+166}$ & $\mathrm{E}$ \\
\hline
\end{tabular}

Note. A: Deming et al. (2011), B: Bakos et al. (2010). C: Gaia Collaboration et al. (2016a). D: Huber et al. (2017), E: This work.

and correspondingly scaling the Hamiltonian by $m_{b} \sqrt{\mathcal{G} M_{\star} a_{b}}$, giving

$$
\begin{aligned}
\mathcal{H}^{\prime}= & \frac{1}{16} n_{b} \frac{m_{c}}{M_{\star}}\left(\frac{a_{b}}{a_{c} \sqrt{1-e_{c}^{2}}}\right)^{3}\left[\frac{\left(5-3 G^{2}\right)\left(3 H^{2}-G^{2}\right)}{G^{2}}\right. \\
& \left.+\frac{15\left(1-G^{2}\right)\left(G^{2}-H^{2}\right) \cos 2 g}{G^{2}}\right]+3 n_{b} \frac{\mathcal{G} M_{\star}}{a_{b} c^{2}} \frac{1}{G} .
\end{aligned}
$$

Here, we have written the expression in terms of the mean motion $n_{b}=\sqrt{\mathcal{G} M_{\star} / a^{3}}$.

The rapid apsidal precession due to GR may suppress the Kozai resonance, which requires a slowly varying $\omega_{b}$. We calculate the GR precession rate,

$$
\dot{\omega}_{G R}=3 n_{b} \frac{\mathcal{G} M_{\star}}{a_{b} c^{2}} \frac{1}{G^{2}} \approx 2.2 \times 10^{-4} \mathrm{yr}^{-1},
$$

which gives a precession period of approximately 30,000 years.
In comparison, the Kozai timescale is given by (Kiseleva et al. 1998)

$$
\tau=\frac{2 P_{c}^{2}}{3 \pi P_{b}^{2}} \frac{M_{\star}}{m_{c}}\left(1-e_{c}^{2}\right)^{3 / 2} \approx 4 \times 10^{5} \text { years },
$$

an order of magnitude longer. Thus, we expect that the Kozai mechanism is suppressed in this system.

To confirm this, we examine the phase space of the Hamiltonian (2). The Hamiltonian admits two integrals of motion: $H$ as well as $\mathcal{H}^{\prime}$ itself. Thus, any given phase-space portrait is parameterized by $H$, which translates to a particular $i_{\max }$, the inclination of the inner planet attained when its orbit is circular. Along level curves of $\mathcal{H}^{\prime}$, the variables $G, g$ trace out trajectories in a two-dimensional phase space, where the eccentricity is given by $e_{b}=\sqrt{1-G^{2}}$, which specifies the instantaneous inclination via the conservation of $H$.

We plot the phase-space portraits for two different values of $i_{\max }$ with and without GR, projected into non-canonical coordinates $e_{b} \cos \omega_{b}$ and $e_{b} \sin \omega_{b}$ in Figure 6. In agreement with the simple timescale argument presented above, we find that the fast precession of $\omega_{b}$ in the HAT-P-11 system is sufficient to suppress Kozai oscillations, such that there is no libration of $e_{b}$ for any value of $i_{\max }$.

We note that simply because the Kozai effect does not operate within the present-day architecture of the HAT-P-11 system does not rule out the possibility that it could have operated previously. This requires the semimajor axis of planet $\mathrm{b}$ to have been larger in the past and to have shrunk to its current configuration due to tidal friction (e.g., Fabrycky \& Tremaine 2007). However, the tidal migration timescale is longer than the tidal circularization timescale by a factor of $1 /\left(1-e^{2}\right)$ (Hut 1981). Thus, tides would tend to circularize the orbit faster than they shrink the orbit. Given that the orbit is still eccentric, we consider it unlikely that tidal damping has shrunk the orbit of HAT-P-11b.

Given the lack of Kozai cycles, we can average out the Kozai term, which leaves us with a trivial dynamical system, governed by a Hamiltonian that only depends on the actions, and thus yields only precession as its consequence. The longitude of ascending node then evolves according to

$$
\begin{aligned}
\frac{d \Omega_{b}}{d t} & =\frac{\partial \mathcal{H}^{\prime}}{\partial H} \\
& =\frac{1}{2} n_{b} \frac{m_{c}}{M_{\star}}\left(\frac{a_{b}}{a_{c} \sqrt{1-e_{c}^{2}}}\right)^{3}\left(\frac{15-9 G^{2}}{G^{2}}\right) H .
\end{aligned}
$$

Thus, $\Omega_{b}$ precesses around the invariant plane defined by the outer planet's orbit, with a period of approximately $3.5 \mathrm{Myr}$, significantly shorter than the age of the system. If the orbit normal of planet $c$ is misaligned with the spin axis of the star by more than half the current observed obliquity of planet $b$, $i_{c} \gtrsim 50^{\circ}$, this would be sufficient to explain HAT-P-11b's approximately polar orbit (Figure 7). However, this does not explain the initial misalignment of HAT-P-11c, which may be the result of planet-planet scattering in the outer system.

We also check that the stellar spin axis does not itself precess more quickly than the inner planet, as such an arrangement would result in a coupling between the star and the inner planet, allowing both to precess together and remain aligned. We follow Spalding \& Batygin (2015) and model the oblateness of the star as a point 
With GR Term
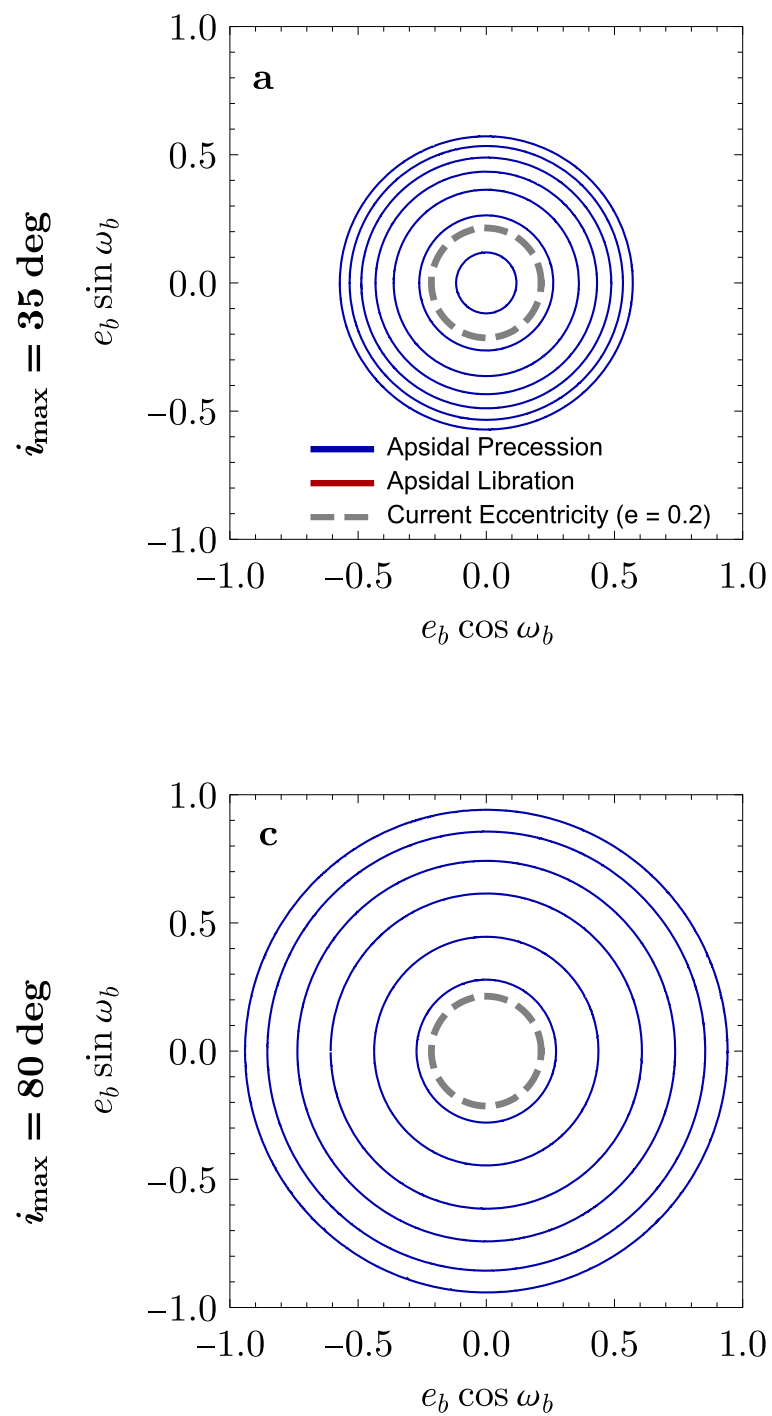

Without GR Term
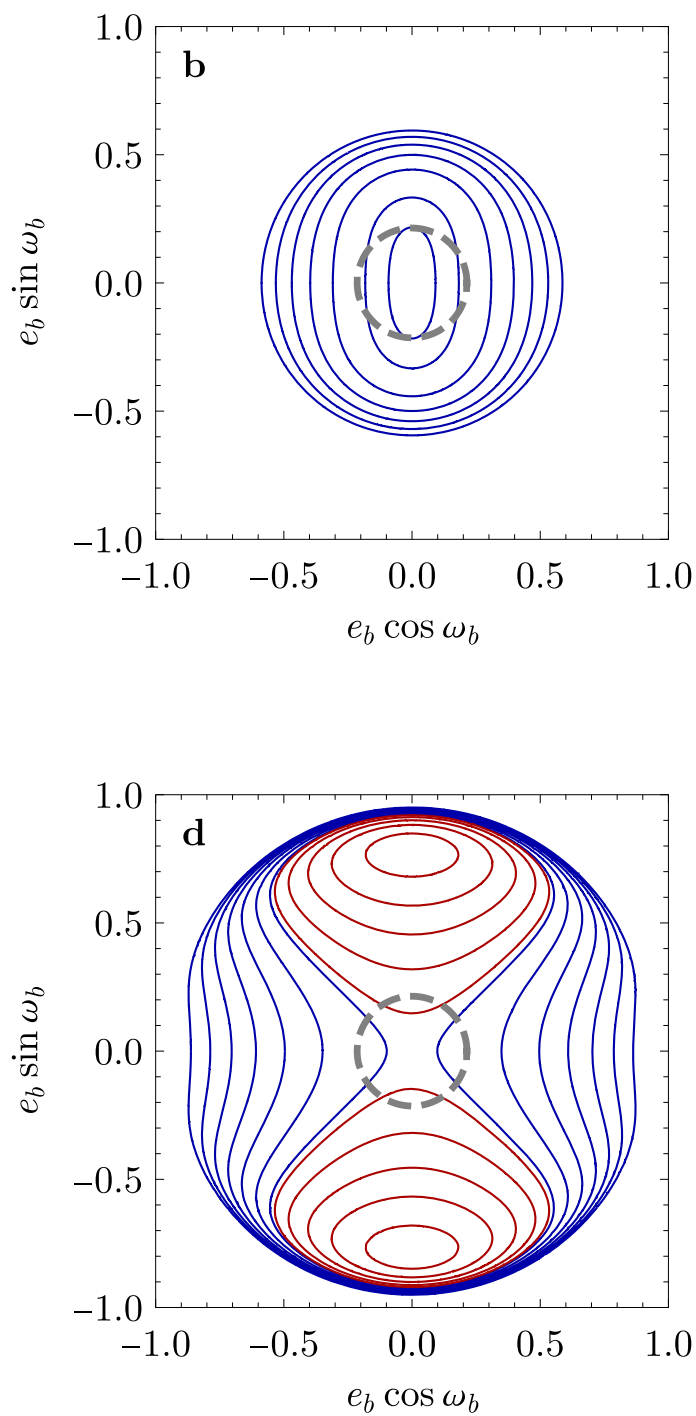

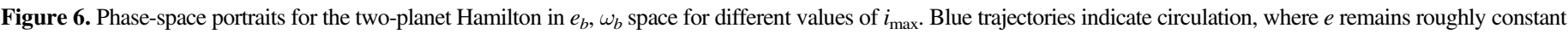

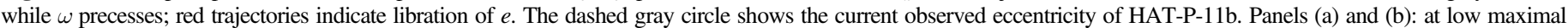

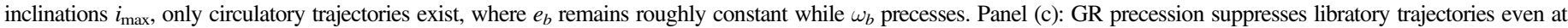

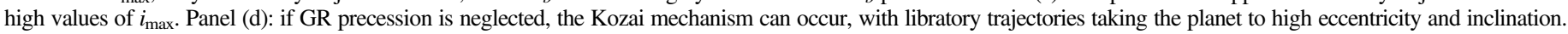

mass surrounded by an orbiting ring with effective semimajor axis

$$
\tilde{a}=\left[\frac{16 \nu^{2} k_{2}^{2} R_{\star}^{6}}{9 I^{2} \mathcal{G} M_{\star}}\right]^{1 / 3} .
$$

Here, $\nu$ is the stellar rotation frequency, and we have used the dimensionless moment of inertia $I=0.08$ and Love number $k_{2}=0.01$. Using Equation (3) for the precession rate due to the torque from the inner planet on the star, we confirm that the stellar precession timescale is on the order of $100 \mathrm{Myr}$, much slower than the precession of the inner planet.

This application of secular theory to the HAT-P-11 system presents a plausible dynamical history that explains the unusual polar orbit of HAT-P-11b. Through precession around the outer planet's orbital plane, HAT-P-11b can attain very high obliquities with respect to the stellar rotation axis, although the angle with respect to the invariant plane remains fixed. A measurement of the mutual inclination between the planetary orbits, for example, through astrometry (Section 7), could help shed more light on this explanation. Nonetheless, irrespective of the exact scenario, this system would have required a large degree of primordial misalignment, either between the orbits of the two planets as described here, or between the stellar spin axis and HAT-P-11b.

\section{The HAT-P-11 System in Context}

Among the planets that have measured obliquities, HAT-P$11 \mathrm{~b}$ is an outlier. It has the smallest planetary to stellar mass ratio and one of the lowest host star effective temperatures for a misaligned planet (Figure 8).

Winn et al. (2010a) first noted a connection between high stellar obliquities and effective temperature, with a higher proportion of planets around hot stars $\left(T_{\text {eff }} \gtrsim 6000 \mathrm{~K}\right)$ with misaligned orbits. They suggested that this could be due to the fact that cool stars have larger convective zones, creating strong tidal coupling with their close-in planets that realigns the star to the planet's orbit normal. In contrast, hot stars without these large convective zones have weaker tidal coupling, leading to a longer tidal realignment timescales. 


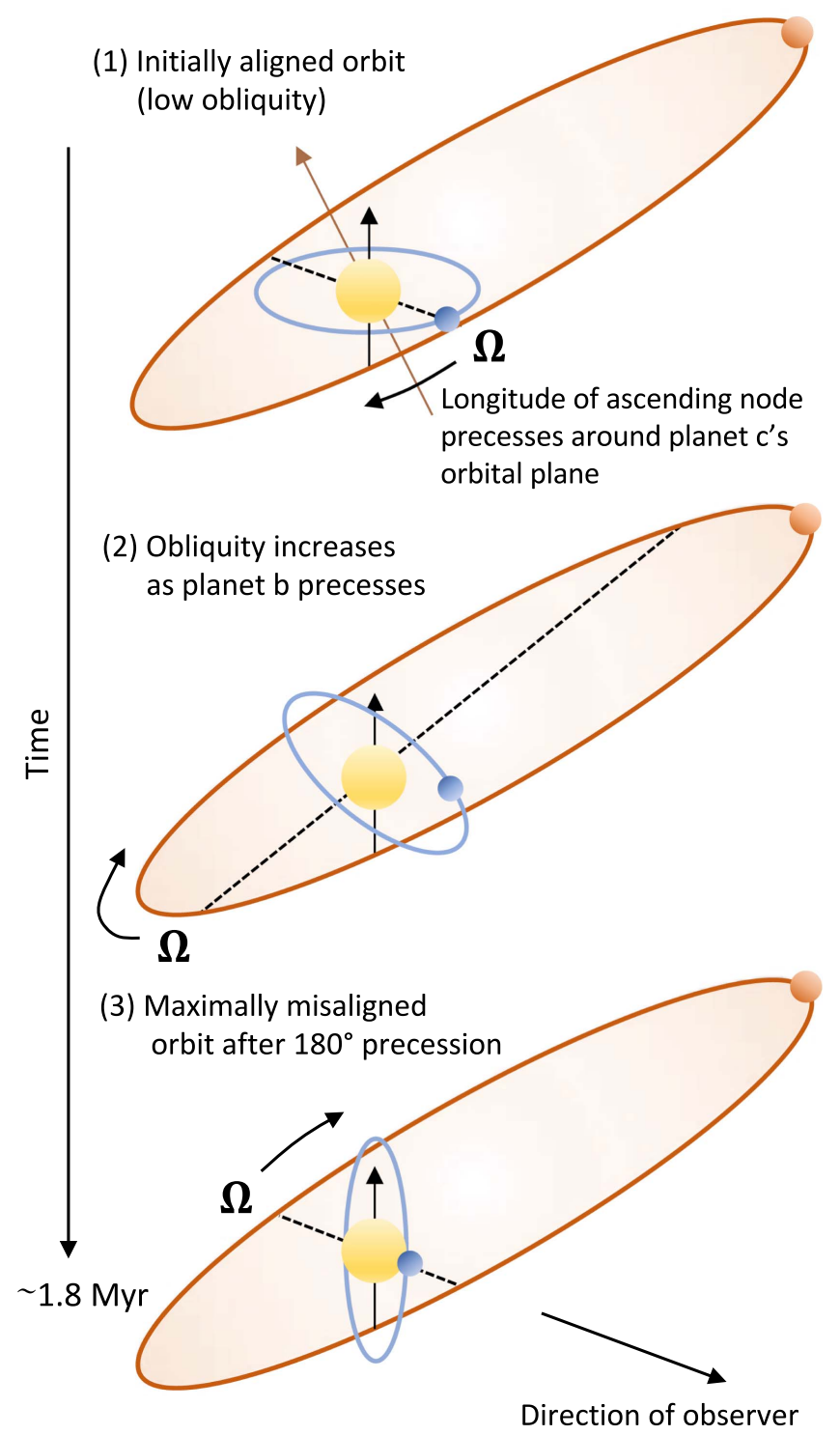

Figure 7. Precession of the longitude of ascending node $\Omega_{b}$ of HAT-P-11b's orbit (blue) around the orbital plane of HAT-P-11c (brown) can result in a polar orbit for planet b. (1) Initially, the inner planet's orbit is aligned with the star's rotation axis (black arrow), but the outer planet has an inclination $\sim 50^{\circ}$ (normal to orbital plane shown as brown arrow). (2) As time progresses, the longitude of ascending node precesses around the plane of the outer planet's orbit, increasing the inclination of the inner planet's orbit relative to the stellar rotation axis. (3) The inner planet reaches a maximum inclination twice that of the outer planet relative to the stellar rotation axis.

Indeed, among the cool stars $\left(T_{\text {eff }}<6000 \mathrm{~K}\right)$ with obliquity measurements, most of the systems exhibiting significant misalignment also have large $a / R_{\star}$ (Figure 8). This corresponds to a longer realignment timescale, allowing systems to retain any primordial inclination. Seen in this light, HAT-P-11b is no longer an outlier, with $a / R_{\star}=16.3 \pm 0.4$. Albrecht et al. (2012) calculated the characteristic realignment timescale for the system to be $\sim 10^{15}$ years, vastly longer than the age of the system. Hence, while the secular precession of HAT-P-11b's orbit normal may be a plausible reason for the observed misalignment, it is not a necessary condition since any primordial misalignment of HAT-P-11b would have also been retained.

While nodal precession is not necessary to maintain HAT-P11b's misalignment for Gyr timescales, it may be for shorter-period planets like HATS-14b (Zhou et al. 2015). Another system for which this mechanism may be at work is WASP-8, which is reminiscent of HAT-P-11 in the following respects: WASP-8 is a cool star with a close-in misaligned planet and a distant giant planet on an eccentric orbit (Knutson et al. 2014).

Nodal precession is likely one of several ways to produce misaligned planets. For example, Kepler-420b (Santerne et al. 2014) and HD 80606b (Hébrard et al. 2010) have stellar companions, suggesting star-planet rather than planet-planet mechanisms. However, if planet-planet nodal precession is a common mechanism to produce misaligned orbits, we predict a correlation between misaligned planets around cool stars and distant, eccentric giants. Such a prediction is testable with future RV, astrometric, or imaging follow-up of known misaligned systems as well as the many more that will soon be discovered by the Transiting Exoplanet Survey Satellite (TESS; Ricker et al. 2014).

\section{Prospects for Future Observations}

\subsection{Secondary Eclipse of HAT-P-11b}

Secondary eclipse observations can provide insight into the albedo and thermal structure of a planet's atmosphere. Huber et al. (2017) reported the detection of the secondary eclipse of HAT-P$11 \mathrm{~b}$ based on Kepler photometry, at an orbital phase of $\phi=0.659$ and depth of $5 \mathrm{ppm}$. Our adopted RV model found an expected secondary eclipse phase of $\phi=0.623_{-0.019}^{+0.018}$, consistent within $2 \sigma$ of the Huber et al. (2017) detection. Future observations with the James Webb Space Telescope (Gardner et al. 2006) should be able to detect and characterize this secondary eclipse.

\subsection{Astrometric Characterization of HAT-P-11c}

The Gaia mission is currently making extremely high-precision astrometric observations of one billion astronomical objects. For HAT-P-11, Gaia is expected to reach an astrometric precision of $\sim 7 \mu$ as (Gaia Collaboration et al. 2016b) by the end of its fiveyear nominal mission.

The wide orbit ( $\left.a=4.13_{-0.16}^{+0.29} \mathrm{au}\right)$ of HAT-P-11c and the system's relative proximity $(37.89 \pm 0.33 \mathrm{pc})$ to Earth means that HAT-P-11c will yield a maximum astrometric signal of $264_{-29}^{+42} \mu$ as. The presence of the planet should in principle be detectable by Gaia during its nominal five-year mission, while a full determination of the orbit's three-dimensional orientation will be possible if the mission duration is extended to 10 years. Although such a measurement will not uniquely determine the mutual inclination of the two planets, it will constrain the difference in angles in a single plane and could help verify the dynamical picture described in Section 5.

\subsection{Direct Imaging of HAT-P-11c}

HAT-P-11c's eccentric orbit takes it up to $6.61_{-0.30}^{+0.52}$ au from its host star. Taking into account the argument of periastron of the orbit, the maximum sky-projected planet-star separation is $134_{-10}^{+15}$ mas.

Assuming a radius and albedo similar to that of Jupiter, the reflected-light contrast ratio of the planet will be $\sim 6 \times 10^{-9}$. This makes HAT-P-11c a potential although challenging target for high-contrast direct imaging studies. For example, the Wide Field Infrared Survey Telescope (Spergel et al. 2015) is expected to have a $10^{-9}$ effective contrast and 100 mas inner working angle, and may be able to characterize HAT-P-11c. 


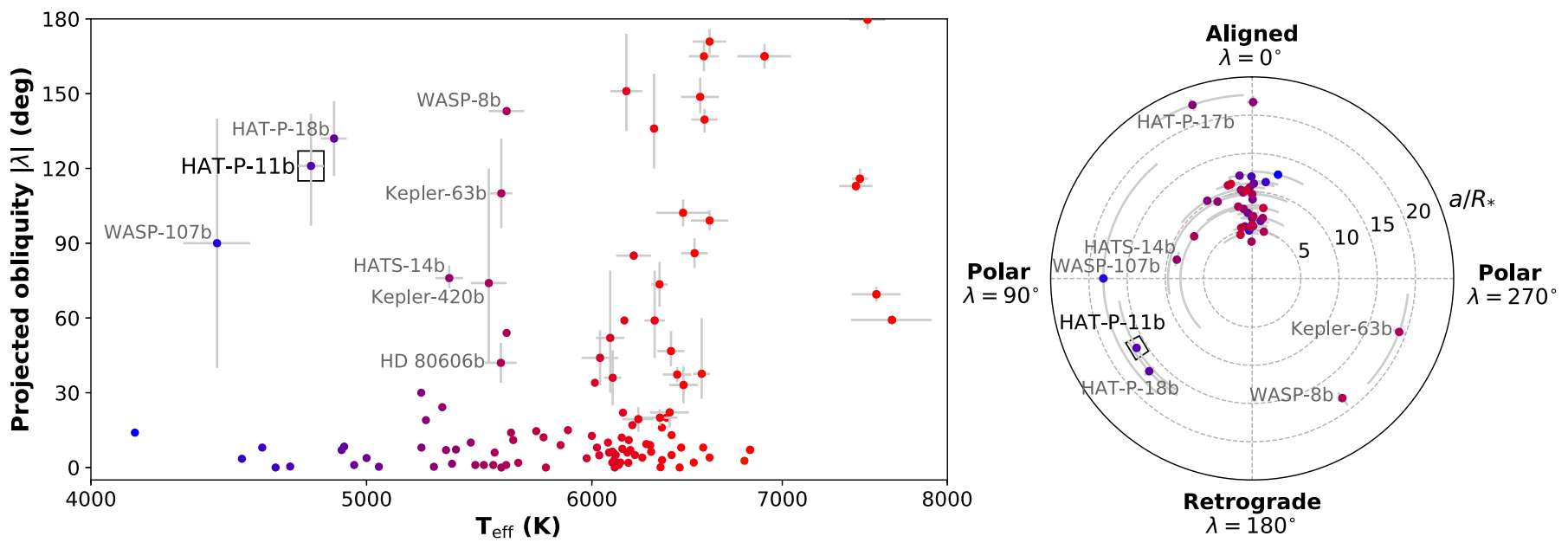

Figure 8. HAT-P-11b in the context of other planets with measured obliquities. (Data compiled from TEPCat as of 2017 October; Southworth 2011; http://www. astro.keele.ac.uk/jkt/tepcat/rossiter.html.) Left: effective temperature and projected stellar obliquities. For clarity, error bars are only shown for planets misaligned by $10^{\circ}$ or more. A large fraction of planets orbiting stars hotter than $\sim 6000 \mathrm{~K}$ are misaligned, perhaps due to the absence of convective envelopes around these stars (see Section 6). HAT-P-11b is one of only a handful of misaligned planets orbiting a star cooler than $6000 \mathrm{~K}$. Right: obliquities for stars with $T_{\text {eff }}<6000 \mathrm{~K}$ as a function of $a / R_{\star}$. Close-in planets tend to be aligned, but this preference vanishes for $a / R_{\star} \gtrsim 15$. The mapping between point color and $T_{\text {eff }}$ is the same as in the left panel.

\section{Conclusion}

The HAT-P-11 system is one of the best-studied exoplanet systems, with long baseline RV and photometric data sets and RM measurements. Here, we extend the RV baseline to 10 years and discover a new $1.5 M_{J}$ giant plant on a distant, eccentric orbit. We found that the presence of HAT-P-11c may explain the previously known misalignment of HAT-P-11b through nodal precession. This mechanism may help to explain the diversity of exoplanet obliquities as a function of orbital distance and host star type. Further characterization of the HAT-P-11 system will soon be possible thanks to upcoming spectroscopic, astrometric, and imaging facilities.

We thank Joshua Winn for helpful discussions that improved the final manuscript. We thank the many observers who contributed to the measurements reported here including M. Bottom, B. Bowler, P. Butler, J. Brewer, J. Crepp, C. Chubak, K. Clubb, I. Crossfield, D. Fischer, E. Ford, E. Gaidos, M. Giguere, J. Johnson, M. Kao, G. Marcy, T. Morton, G. Mandushev, K. Peek, S. Pineda, G. Torres, S. Vogt, P. Worden, M. Zhao.

S.W.Y. acknowledges support from the Caltech Marcella Bonsall Summer Undergraduate Research Fellowship. E.A.P. acknowledges support from a Hubble Fellowship grant HST-HF251365.001-A awarded by the Space Telescope Science Institute, which is operated by the Association of Universities for Research in Astronomy, Inc. for NASA under contract NAS 5-26555. The data presented herein were obtained at the W. M. Keck Observatory, which is operated as a scientific partnership among the California Institute of Technology, the University of California and the National Aeronautics and Space Administration. The Observatory was made possible by the generous financial support of the W. M. Keck Foundation. The authors wish to recognize and acknowledge the very significant cultural role and reverence that the summit of Maunakea has long had within the indigenous Hawaiian community. We are most fortunate to have the opportunity to conduct observations from this mountain.

Software: Numpy/Scipy (Van Der Walt et al. 2011), Matplotlib (Hunter 2007), Pandas (McKinney 2010), Astropy (Astropy Collaboration et al. 2013), emcee
(Goodman \& Weare 2010; Foreman-Mackey et al. 2013), RadVel (Fulton et al. 2018a).

Facility: Keck:I (HIRES).

\section{Appendix A Model Comparison and Selection}

In Section 4, we adopted an RV model incorporating the effect of two planets and an activity-induced signal. This model was chosen after exploring two other possibilities: (1) a single-planet model where the long-term RV trend must be completely accounted for by the activity-RV correlation, and (2) a two-planet model without any activity-RV correlation. Model (3) is the twoplanet plus activity model. To compare the models, we compute the BIC, which incorporates the log-likelihood of the model and a penalty for the number of free parameters. A model with lower BIC is preferred, with $|\Delta \mathrm{BIC}| \gtrsim 10$ being strongly favored. We present the results of the RV fits in Table 3.

We found that the single-planet model (1) gave the poorest fit, with rms residuals of almost $17 \mathrm{~m} \mathrm{~s}^{-1}$ and a high BIC. Models (2) and (3) provided a significant improvement in the rms residuals, indicating that the long-period signal can be best explained by the presence of an outer planet. The model parameters found by both models for the two planets are similar, typically differing by less than $1 \sigma$. However, model (3) had the lowest BIC $(\Delta \mathrm{BIC}=-16)$ despite the additional model complexity. This is in accordance with our conclusions from Section 3, where we saw that stellar activity does account for some, but not all, of the RV variation. The BIC analysis thus supports our choice of a two-planet RV model with a linear activity-RV correction.

We also investigated more complex models to determine if there were additional planets in the system. We performed an iterative search using the 2DKLS periodogram (O'Toole et al. 2009) following the technique of Fulton et al. (2015) and Howard \& Fulton (2016). An empirical false-alarm probability (eFAP) is calculated from a histogram of periodogram amplitudes.

Figure 9 shows the periodogram for a three- versus twoplanet model. We find no significant peaks above the eFAP threshold of $1 \%$, with only minor peaks close to the stellar rotation period of 29 days and its aliases. Thus, inclusion of a third planet is not justified given the current data set. 


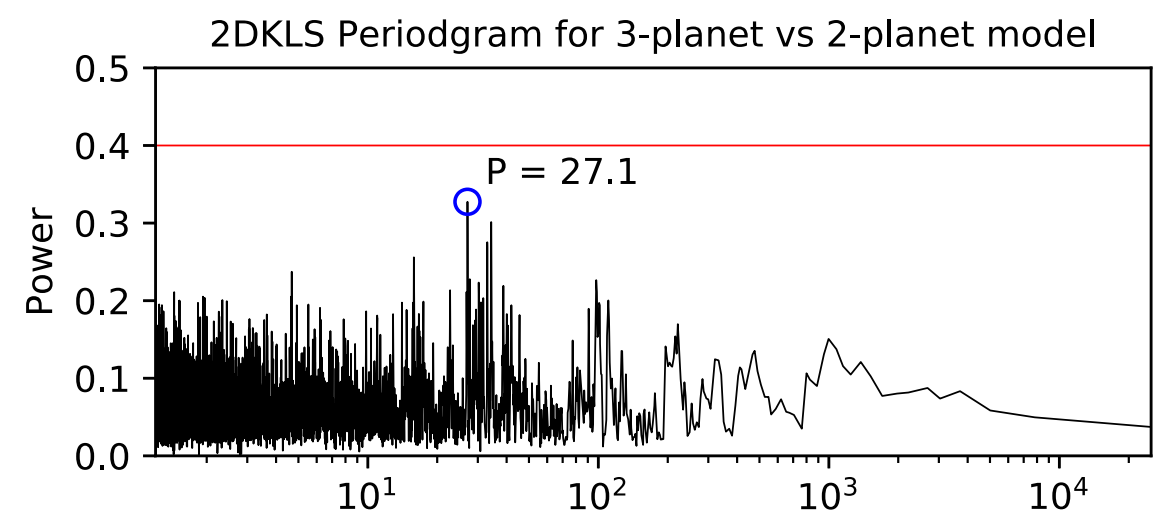

Figure 9. Two-dimensional Keplerian Lomb-Scargle (2DKLS) periodogram where a third Keplerian is fit to the residuals after removing HAT-P-11b and c. No additional significant periodic signals could be detected.

Table 3

Comparison of RV-Activity Models

\begin{tabular}{lccc}
\hline \hline & Model 1 & Model 2 & $\begin{array}{c}\text { Model 3 } \\
\text { (Adopted) }\end{array}$ \\
\hline Number of planets & 1 & 2 & 2 \\
$S_{\text {HK correction }}$ & Yes & No & Yes \\
Number of free parameters & 6 & 10 & 11 \\
\hline
\end{tabular}

Planet b parameters

$P_{b}$ (days)

$T \operatorname{conj}_{b}(\mathrm{JD})$

$e_{b}$

$\omega_{b}\left({ }^{\circ}\right)$

$K_{b}\left(\mathrm{~m} \mathrm{~s}^{-1}\right)$

$\equiv 4.887802443$

$\equiv 2454957.8132067$

Planet c parameters

$P_{c}$ (days)

$T \operatorname{conj}_{c}$ (JD)

$e_{c}$

$\omega_{c}\left({ }^{\circ}\right)$

$K_{c}\left(\mathrm{~m} \mathrm{~s}^{-1}\right)$

$0.223_{-0.034}^{+0.036}$
$18_{-17}^{+15}$

$0.218_{-0.031}^{+0.034}$

$\begin{array}{ccc}0.19_{-0.13}^{+0.14} & 0.223_{-0.034}^{+0.036} & 0.218_{-0.031}^{+0.034} \\ 33_{-57}^{+44} & 18_{-17}^{+15} & 19_{-16}^{+14} \\ 10.0_{-2.3}^{+2.2} & 10.51_{-0.71}^{+0.72} & 10.42_{-0.66}^{+0.64}\end{array}$

Global parameters

$c_{S}\left(\mathrm{~m} \mathrm{~s}^{-1} S_{\mathrm{HK}}{ }^{-1}\right)$

$\gamma\left(\mathrm{m} \mathrm{s}^{-1}\right)$

$\sigma_{\mathrm{jit}}\left(\mathrm{m} \mathrm{s}^{-1}\right)$

\begin{tabular}{lcc}
$\ldots$ & $3335_{-160}^{+280}$ & $3407_{-190}^{+360}$ \\
$\cdots$ & $2456739_{-39}^{+30}$ & $2456746_{-32}^{+24}$ \\
$\cdots$ & $0.560_{-0.035}^{+0.034}$ & $0.601_{-0.031}^{+0.032}$ \\
$\cdots$ & $140.0_{-4.9}^{+4.8}$ & $143.7_{-4.9}^{+4.8}$ \\
$\cdots$ & $32.1 \pm 1.4$ & $30.9 \pm 1.3$ \\
\hline
\end{tabular}

Model comparison

rms residuals $\left(\mathrm{m} \mathrm{s}^{-1}\right)$

BIC

$\begin{array}{cc}92_{-44}^{+43} & \cdots \\ -10.2 \pm 1.5 & 0.223_{-0.034}^{+0.036} \\ 17.3_{-1.0}^{+1.1} & 5.40_{-0.34}^{+0.38}\end{array}$

$78.6_{-16.1}^{+16.8}$

$-1.80_{-0.79}^{+0.84}$

$4.98_{-0.32}^{+0.36}$

Note. $c_{S}$ is the slope of the linear correlation between RV and $S_{\mathrm{HK}}$.

\section{Appendix B}

Comparison with HD 99492

To validate the joint RV-activity methodology described in Section 4, we applied the same analysis to the HD 99492 system, another moderately active mid-K dwarf with a decade of RV measurements. Similar to HAT-P-11, HD 99492 has a relatively short-period ( $P=17.1$ days) inner planet first discovered by Marcy et al. (2005). Meschiari et al. (2011) later attributed a 5000 day RV signal to a distant giant planet. However, the star's activity cycle, as traced by the $S_{\mathrm{HK}}$ index, was found to have similar periodicity by Kane et al. (2016), who subtracted the effect of the inner planet from the RVs and found a strong correlation between these residual RVs and the $S_{\mathrm{HK}}$ values. They used this to argue that the outer planet reported by Meschiari et al. (2011) should therefore be attributed to stellar activity.
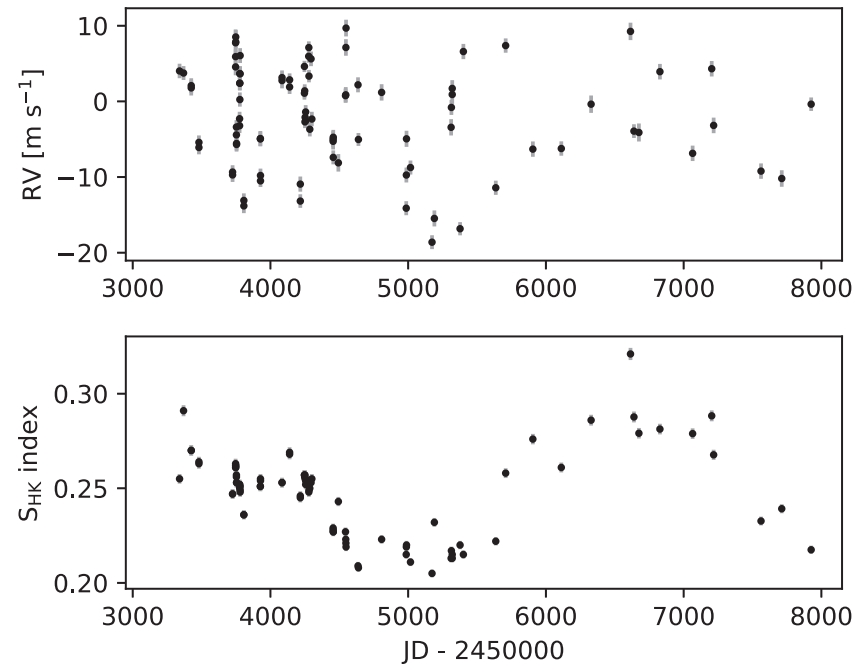

Figure 10. RV measurements (top) and $S_{\mathrm{HK}}$ indices (bottom) for HD 99492. Similar to HAT-P-11, the $S_{\mathrm{HK}}$ index exhibits a long-term variation indicative of an activity cycle.

Table 4

Model Comparison for HD 99492

\begin{tabular}{lccc}
\hline \hline & $\begin{array}{c}\text { Model 1 } \\
\text { (Adopted) }\end{array}$ & Model 2 & Model 3 \\
\hline$n_{\text {planets }}$ & 1 & 2 & 2 \\
$S_{\text {HK }}$ correction & Full & None & Full \\
\hline rms residuals $\left(\mathrm{m} \mathrm{s}^{-1}\right)$ & 3.61 & 3.69 & 3.39 \\
BIC & 502.98 & 524.53 & 513.52 \\
\hline
\end{tabular}

We investigated the HD 99492 system using the same joint activity-RV analysis described previously. We used 89 HIRES spectra taken by the CPS program over 13 years, from 2004 to 2017 (Figure 10). A long-term periodic signal can clearly be seen in the $S_{\mathrm{HK}}$ measurements, of almost identical period and phase to the RV variability.

We then fit these RV measurements using three different models, similar to those used for fitting HAT-P-11: (1) a singleplanet model with activity-RV decorrelation; (2) a two-planet model without any decorrelation; and (3) a two-planet model with activity-RV decorrelation. We compare these three models in Table 4. 
For the HD 99492 system, the lowest BIC was achieved for the model with only one planet, with the residual RV signal fully accounted for by stellar activity $(\triangle \mathrm{BIC}=-11)$. The slope of the $\mathrm{RV}-S_{\mathrm{HK}}$ correlation was found to be $c_{1}=92 \pm 18 \mathrm{~m} \mathrm{~s}^{-1} S_{\mathrm{HK}}{ }^{-1}$, so the semi-amplitude of the activity-induced RV signal is $\sim 5 \mathrm{~m} \mathrm{~s}^{-1}$, similar to that found for HAT-P-11. We also noted that when fitting two planets with activity decorrelation (model 3), an MCMC analysis found the RV semi-amplitude of the outer planet to be $1.8_{-2.0}^{+1.2} \mathrm{~m} \mathrm{~s}^{-1}$, not significantly different from zero. Finally, a Keplerian fit to the $S_{\mathrm{HK}}$ time series found a cycle whose period and phase are consistent with the fit to the RV time series within $1 \sigma$, suggesting that the two signals are indeed correlated.
Here, we see that the same analysis as previously applied to HAT-P-11 now readily rejects the presence of an outer planet in the HD 99492 system and supports the integrity of our methodology.

\section{Appendix C}

\section{Posterior Distribution of RV Model Parameters}

We provide in Figure 11 the posterior distributions for each of the model parameters. To derive these distributions, we explored the likelihood surface with an MCMC analysis.

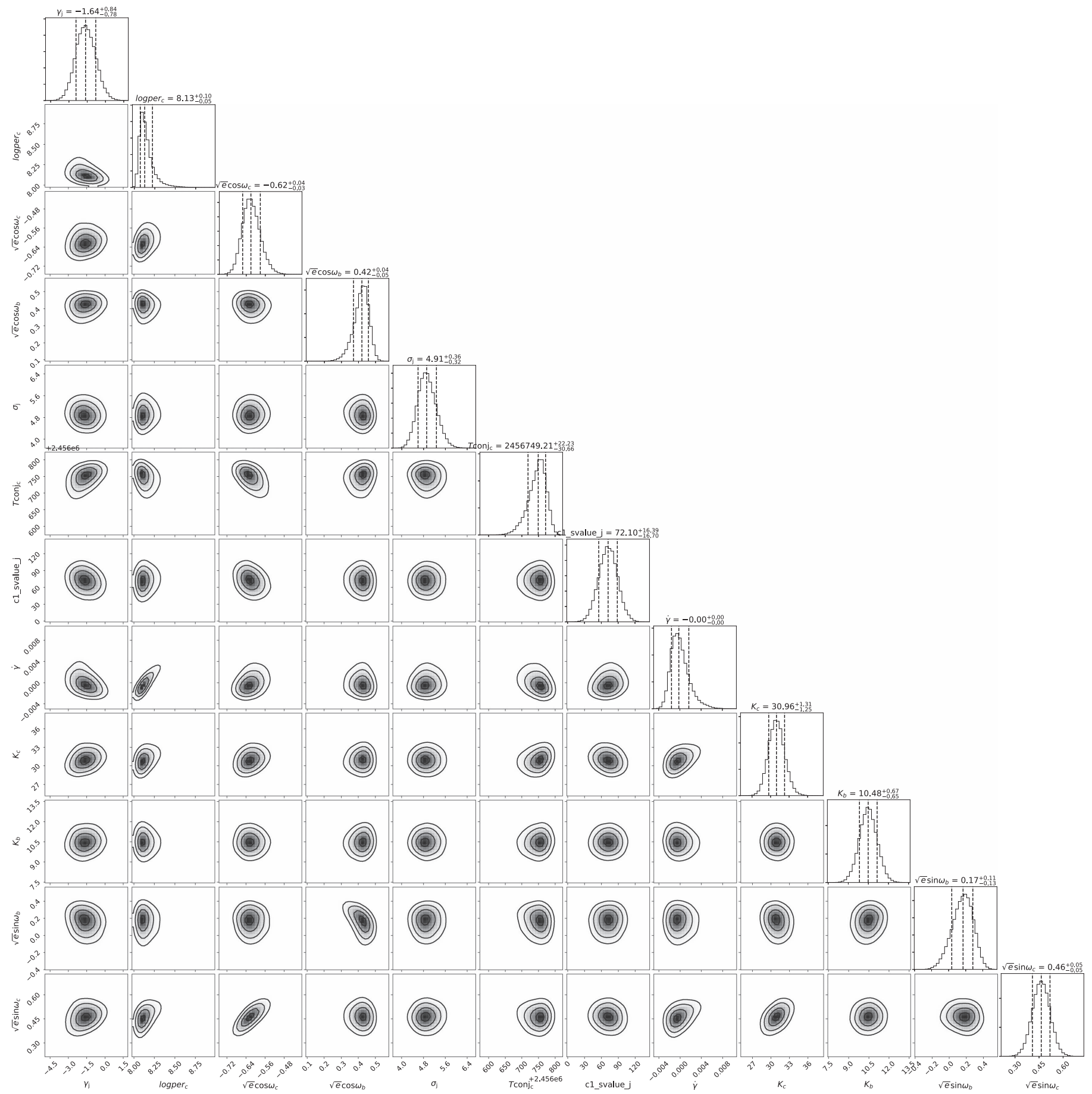

Figure 11. Corner plot showing posterior distributions for each model parameter. The first plot in each column shows the single-variable distribution, with the vertical dashed lines denoting the most probable value and $1 \sigma$ confidence bounds. The remaining plots show joint distributions between each pair of model parameters. 


\section{ORCID iDs}

Samuel W. Yee 주 https://orcid.org/0000-0001-7961-3907

Erik A. Petigura (i) https://orcid.org/0000-0003-0967-2893

Benjamin J. Fulton (1) https://orcid.org/0000-0003-3504-5316

Gáspár Á. Bakos ำ https://orcid.org/0000-0001-7204-6727

Joel D. Hartman (1) https://orcid.org/0000-0001-8732-6166

Lea A. Hirsch (1) https://orcid.org/0000-0001-8058-7443

Andrew W. Howard (10) https://orcid.org/0000-0001-

8638-0320

Howard Isaacson iㅏ https://orcid.org/0000-0002-0531-1073

Molly R. Kosiarek (i) https://orcid.org/0000-0002-6115-4359

Evan Sinukoff (i) https://orcid.org/0000-0002-5658-0601

Lauren M. Weiss (i) https://orcid.org/0000-0002-3725-3058

\section{References}

Albrecht, S., Winn, J. N., Johnson, J. A., et al. 2012, ApJ, 757, 18 Astropy Collaboration, Robitaille, T. P., Tollerud, E. J., et al. 2013, A\&A, 558, A33

Bakos, G. Á, Torres, G., Pál, A., et al. 2010, ApJ, 710, 1724

Batygin, K. 2012, Natur, 491, 418

Borucki, W. J., Koch, D., Basri, G., et al. 2010, Sci, 327, 977

Dai, F., \& Winn, J. N. 2017, AJ, 153, 205

Deming, D., Sada, P. V., Jackson, B., et al. 2011, ApJ, 740, 33

Dumusque, X., Boisse, I., \& Santos, N. C. 2014, ApJ, 796, 132

Eastman, J., Gaudi, B. S., \& Agol, E. 2013, PASP, 125, 83

Fabrycky, D., \& Tremaine, S. 2007, ApJ, 669, 1298

Ford, E. B., Kozinsky, B., \& Rasio, F. A. 2000, ApJ, 535, 385

Foreman-Mackey, D., Hogg, D. W., Lang, D., \& Goodman, J. 2013, PASP, 125,306

Fulton, B., Blunt, S., Petigura, E., et al. 2018a, California-Planet-Search/ radvel: Version 1.1.0, Zenodo, doi:10.5281/zenodo.1143674

Fulton, B. J., Petigura, E. A., Blunt, S., \& Sinukoff, E. 2018b, arXiv:1801. 01947

Fulton, B. J., Weiss, L. M., Sinukoff, E., et al. 2015, ApJ, 805, 175

Gaia Collaboration, Brown, A. G. A., Vallenari, A., et al. 2016a, A\&A, 595, A2

Gaia Collaboration, Prusti, T., de Bruijne, J. H. J., et al. 2016b, A\&A, 595, A1

Gardner, J. P., Mather, J. C., Clampin, M., et al. 2006, SSRv, 123, 485

Gomes da Silva, J., Santos, N. C., Bonfils, X., et al. 2011, A\&A, 534, A30

Goodman, J., \& Weare, J. 2010, Communications in Applied Mathematics and Computational Science, 5, 65

Haywood, R. D., Collier Cameron, A., Queloz, D., et al. 2014, MNRAS, 443, 2517
Hébrard, G., Désert, J.-M., Díaz, R. F., et al. 2010, A\&A, 516, A95

Hirano, T., Narita, N., Shporer, A., et al. 2011, PASJ, 63, 531

Howard, A. W., \& Fulton, B. J. 2016, PASP, 128, 114401

Howard, A. W., Johnson, J. A., Marcy, G. W., et al. 2010, ApJ, 721, 1467

Huber, K. F., Czesla, S., \& Schmitt, J. H. M. M. 2017, A\&A, 597, A113

Hunter, J. D. 2007, CSE, 9, 90

Hut, P. 1981, A\&A, 99, 126

Isaacson, H., \& Fischer, D. 2010, ApJ, 725, 875

Kane, S. R., Thirumalachari, B., Henry, G. W., et al. 2016, ApJL, 820, L5

Kaula, W. M. 1964, RvGSP, 2, 661

Kipping, D. M. 2013, MNRAS, 434, L51

Kiseleva, L. G., Eggleton, P. P., \& Mikkola, S. 1998, MNRAS, 300, 292

Knutson, H. A., Fulton, B. J., Montet, B. T., et al. 2014, ApJ, 785, 126

Lovis, C., Dumusque, X., Santos, N. C., et al. 2011, arXiv:1107.5325

Marcy, G. W., \& Butler, R. P. 1992, PASP, 104, 270

Marcy, G. W., Butler, R. P., Vogt, S. S., et al. 2005, ApJ, 619, 570

Mardling, R. A. 2010, MNRAS, 407, 1048

McKinney, W. 2010, in Proc. 9th Python in Science Conf., ed. S. van der Walt \& J. Millman, 51

Meschiari, S., Laughlin, G., Vogt, S. S., et al. 2011, ApJ, 727, 117

Morris, B. M., Hawley, S. L., Hebb, L., et al. 2017a, ApJ, 848, 58

Morris, B. M., Hebb, L., Davenport, J. R. A., Rohn, G., \& Hawley, S. L. 2017b, ApJ, 846, 99

Nagasawa, M., Ida, S., \& Bessho, T. 2008, ApJ, 678, 498

O’Toole, S. J., Tinney, C. G., Jones, H. R. A., et al. 2009, MNRAS, 392 641

Ricker, G. R., Winn, J. N., Vanderspek, R., et al. 2014, Proc. SPIE, 9143, 914320

Robertson, P., Mahadevan, S., Endl, M., \& Roy, A. 2014, Sci, 345, 440

Rogers, T. M., Lin, D. N. C., \& Lau, H. H. B. 2012, ApJL, 758, L6

Sanchis-Ojeda, R., \& Winn, J. N. 2011, ApJ, 743, 61

Santerne, A., Hébrard, G., Deleuil, M., et al. 2014, A\&A, 571, A37

Schwarz, G. 1978, AnSta, 6, 461

Shine, R. A., \& Linsky, J. L. 1974, SoPh, 39, 49

Southworth, J. 2011, MNRAS, 417, 2166

Spalding, C., \& Batygin, K. 2015, ApJ, 811, 82

Spergel, D., Gehrels, N., Baltay, C., et al. 2015, arXiv:1503.03757

Valenti, J. A., Butler, R. P., \& Marcy, G. W. 1995, PASP, 107, 966

Van Der Walt, S., Colbert, S. C., \& Varoquaux, G. 2011, arXiv:1102.1523

Vaughan, A. H., Preston, G. W., \& Wilson, O. C. 1978, PASP, 90, 267

Vogt, S. S., Allen, S. L., Bigelow, B. C., et al. 1994, Proc. SPIE, 2198, 362

Winn, J. N., Fabrycky, D., Albrecht, S., \& Johnson, J. A. 2010a, ApJL, 718, L145

Winn, J. N., Johnson, J. A., Howard, A. W., et al. 2010b, ApJL, 723, L223

Zechmeister, M., \& Kürster, M. 2009, A\&A, 496, 577

Zhou, G., Bayliss, D., Hartman, J. D., et al. 2015, ApJL, 814, L16 